\title{
Cardiac echinococcosis secondary to hepatic echinococcosis: a rare case report
}

\author{
Ran Zhang^, Can Shen, Li Rao^ \\ Department of Cardiology, West China Hospital of Sichuan University, Chengdu, China \\ Correspondence to: Li Rao, MD. Department of Cardiology, West China Hospital of Sichuan University, No. 37 Guo Xue Xiang, Chengdu, China. \\ Email: lrlz1989@163.com.
}

\begin{abstract}
Cystic echinococcosis (CE) is a zoonotic parasitic infection, which is very rare in developed countries. It can affect all internal organs, while cardiac echinococcosis is extremely rare, especially in children. Slowly enlarging hydatid cyst usually remains asymptomatic until the size or space occupying effects the involved organ and induces symptoms. The progression of cardiac echinococcosis can be very hidden, and the symptoms are similar to that of other cardiovascular diseases, which raises the difficulty in accurate diagnosis. We present a 13-year-old young girl with a history of hepatic echinococcosis who developed a huge cardiac hydatid cyst, but her symptoms were not specific, while the physical tests and biochemical examinations were unremarkable. Her residential area in Tibet and previous medical history of hepatic echinococcosis gave us clues in the diagnosis of cardiac echinococcosis. Combined with computed tomography (CT) and magnetic resonance imaging (MRI), the cardiac echinococcosis was finally confirmed, and the cardiac symptoms were relieved after surgical removal of the cardiac hydatid cyst. This is the first report of children's cardiac echinococcosis secondary to hepatic echinococcosis, and it remarks on the importance of rapid consideration of cardiac echinococcosis even if no remarkable symptoms or indexes are present. Moreover, the combination of previous history and imaging techniques are indispensable for obtaining a definite diagnosis.
\end{abstract}

Keywords: Cardiac echinococcosis; hepatic echinococcosis; cystic echinococcosis (CE); pediatric cardiac echinococcosis; case report

Submitted Sep 02, 2021. Accepted for publication Jan 13, 2022.

doi: $10.21037 / \mathrm{cdt}-21-569$

View this article at: https://dx.doi.org/10.21037/cdt-21-569

\section{Introduction}

Cystic echinococcosis (CE) is a zoonotic parasitic disease that is epidemic in many parts of the world, especially in livestock-raising areas (1). CE can occur almost anywhere in the human body (2). Hepatic echinococcosis is the most common type that accounts for $74.8 \%$ of CE. But cardiac and pericardial echinococcosis are extremely rare, especially in children, which only account for $0.02-2.0 \%$ of the total incidence rate of echinococcosis (3).

The clinical manifestation of CE varies according to the number and size of cysts, growth stage, associated complications, and affected tissue (4). The formation of hydatid cysts needs $1-5$ years generally, and symptoms usually present in individuals over 20 years (5), thus cardiac echinococcosis of children is an extremely rare condition. Most of the symptoms of cardiac echinococcosis are similar to those of other cardiovascular diseases, such as cardiomyopathy, pulmonary hypertension, and myocardial infarction (6). Therefore, the early diagnosis of cardiac echinococcosis is difficult. Clinicians should be aware of the potential cardiac manifestations of the parasitic diseases in both immunocompetent and immunocompromised patients,

^ ORCID: Ran Zhang, 0000-0002-4458-8454; Li Rao, 0000-0003-2555-095X. 


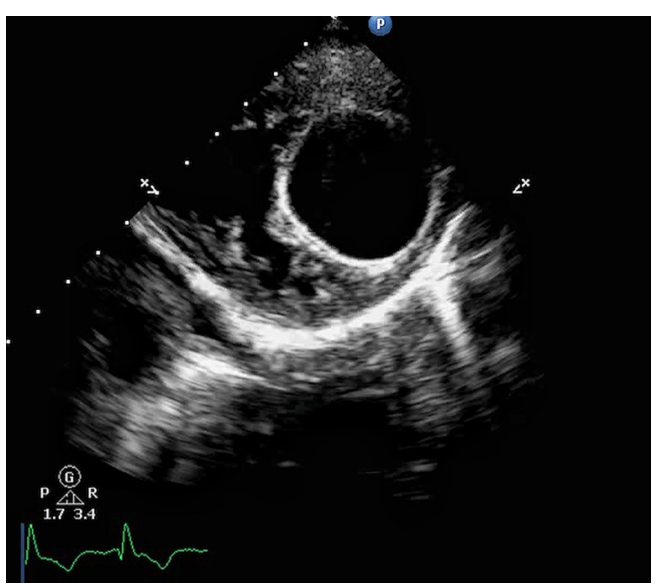

Figure 1 TTE showed a giant cyst within the left ventricle. TTE, transthoracic echocardiography.

and the residence, contact history and past medical history are important to obtain a rapid and accurate diagnosis.

Cardiac echinococcosis is extremely rare. Few reports mentioned cardiac echinococcosis in children $(5,7,8)$. According to the available literature, no case of pediatric cardiac echinococcosis secondary to hepatic echinococcosis has been reported previously. We firstly report a case of pediatric cardiac echinococcosis, which was secondary to the rupture of the primary germinative membrane of cysts located at the liver, and briefly discuss the diagnosis and treatment of cardiac echinococcosis. We present the following case in accordance with the CARE reporting checklist (available at https://cdt.amegroups.com/article/ view/10.21037/cdt-21-569/rc).

\section{Case presentation}

A 13-year-old girl of Chinese Tibetan nationality initially presented with precordial pain and discomfort of the left upper abdomen 3 years ago. She was diagnosed with the 'hepatic cyst' at a local hospital, and surgical removal of the hepatic cyst was performed. But a few months after the operation, the patient suffered from repeated chest pain, which occurred once every 2 or 3 days. The degree of precordial pain was gradually aggravated. Then she was admitted to the Cardiology Department of our hospital. Preliminary echocardiography was performed, and the result showed a cystic occupation within her left ventricle (Figure 1).

The growth and development of the patient were slightly worse than that of her peers. She was living in Tibet plateau areas since childhood. She had no history of hepatitis, tuberculosis, or other infections. The New York Heart Association (NYHA) classification of cardiac function was II. Vitals on admission were notable for blood pressure at $96 / 53 \mathrm{mmHg}$, and a heart rate of 72 beats per minute. No cardiac murmur was heard. The electrocardiograph (ECG) showed normal sinus rhythm. Her physical tests, serologic test (hydatid cyst antibody), enzyme-linked immunosorbent assay (ELISA) and other biochemical examinations were unremarkable.

The computed tomography (CT) was performed. Multiple cysts could be detected at the left lobe of the liver with a maximum diameter of $12.0 \mathrm{~cm} \times 10.4 \mathrm{~cm}$. The capsule of these cysts showed obvious enhancement. A heterogeneous patchy mass with slight enhancement could be found at the right lobe of the liver, and a high-density shadow was noted within this patchy mass, which could be considered as calcification. The tissue surrounding these cysts was obviously compressed. A cystic mass could be detected at the left ventricle. The cyst had a thick capsule wall with obvious enhancement, which was suggestive of parasitic infection (Figure 2). Cardiac magnetic resonance imaging (MRI) confirmed the location of the mass at the left ventricle wall (Figure 3). Combined with her residence, previous hepatic cysts history, imaging findings, and clinical manifestations, cardiac echinococcosis secondary to the hepatic echinococcosis was diagnosed. Therefore, she was transferred to the department of cardiac surgery for resection of the cardiac cyst.

The resection surgery was performed under general anesthesia a few days later. Cardiac dilation and thickening of the left ventricle wall were observed during the surgery. A $27 \mathrm{~mm} \times 28 \mathrm{~mm}$ round cyst was found at the mitral valve plan within the left ventricle. The cyst was composed of inner and outer cystic capsule structures, and it was resected completely. The operation was successful and postoperative transesophageal echocardiography showed no abnormity of the heart valves. Then she returned to the pediatric intensive care units (ICU) for observation. The patient recovered and was in a good general condition. Her hepatic CE also required surgical treatment 3 months after the cardiac cyst resection. Anti-echinococcal drug, albendazole, was administrated with a dosage of $200 \mathrm{mg} / \mathrm{day}$ for 3 months. No adverse and unanticipated events happened, then this patient was subsequently discharged. The historical and current information from onset to the treatment of this patient were summarized in a timeline (Table 1). 

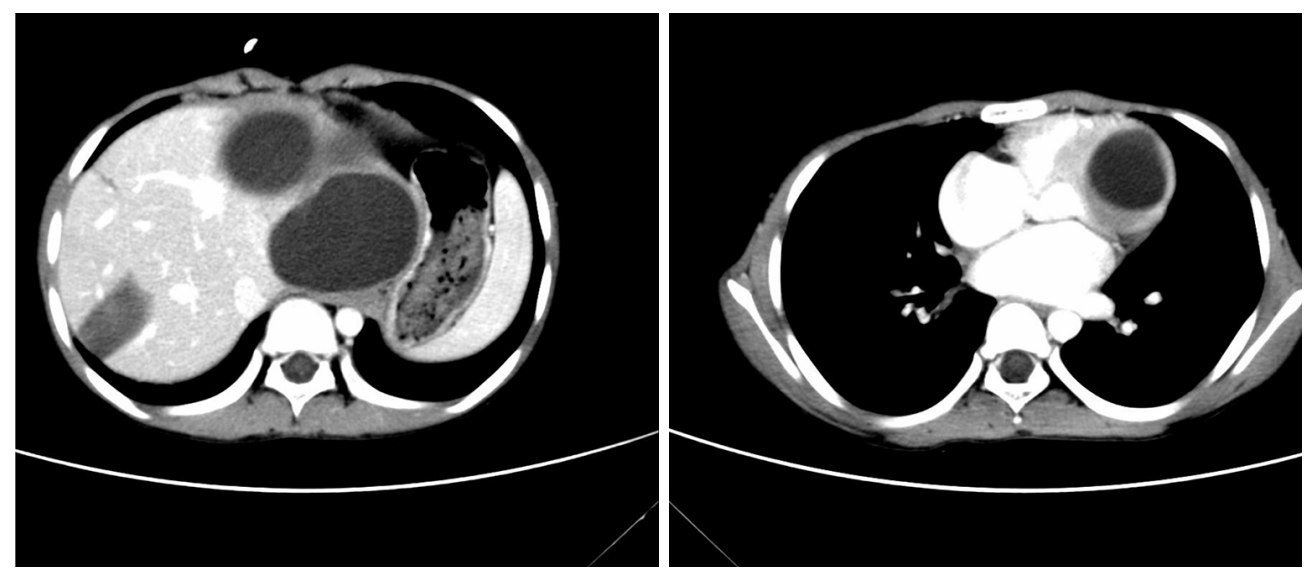

Figure 2 CT showed multiples cysts at liver and a cyst at left ventricle wall. CT, computed tomography.
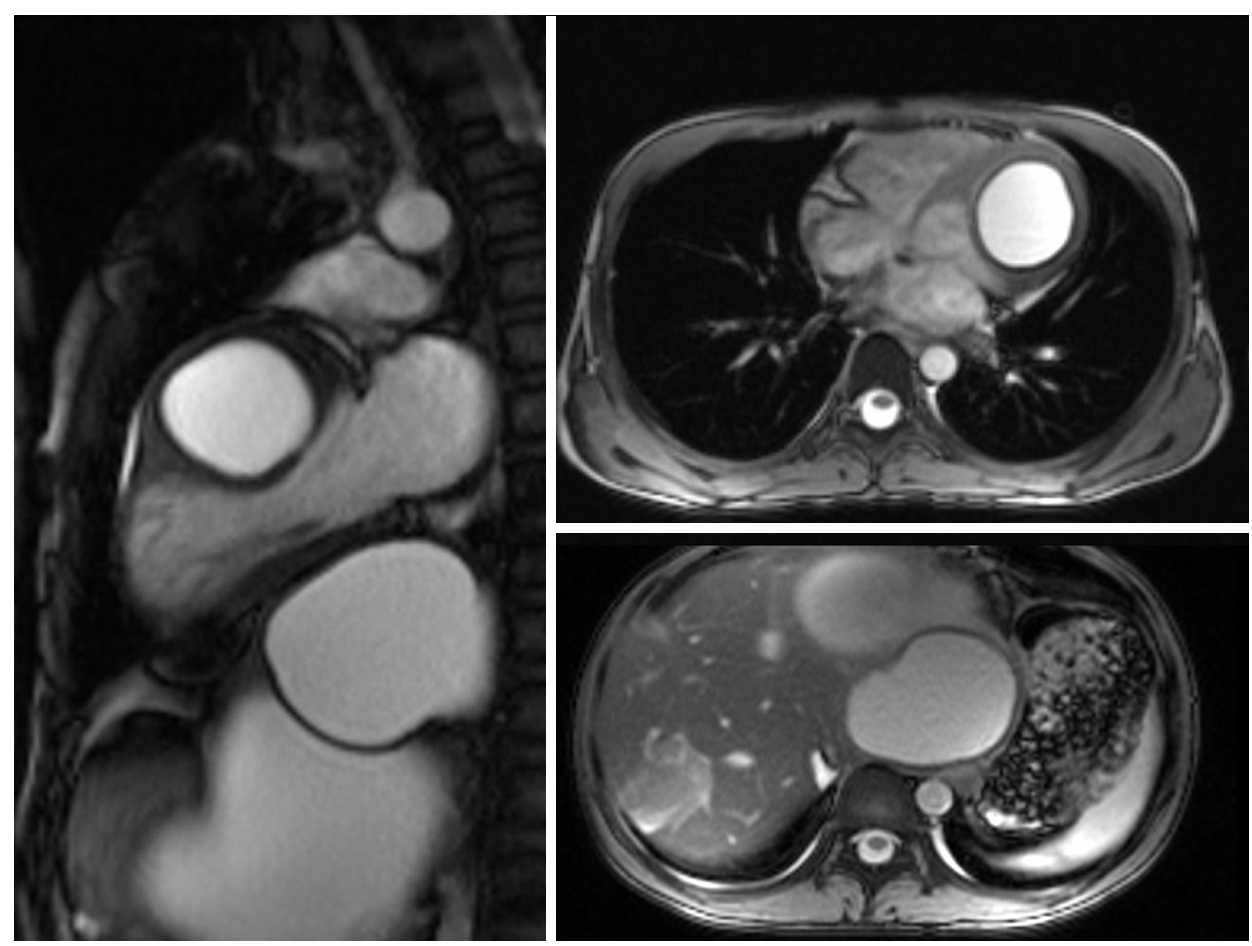

Figure 3 MRI showed the cyst at left ventricle wall in cross and sagittal sections. MRI, magnetic resonance imaging.

All procedures performed in studies involving human participants were in accordance with the ethical standards of the institutional and national research committee and with the Helsinki Declaration (as revised in 2013). Written informed consent was obtained from the patient for publication of this case report and accompanying images. A copy of the written consent is available for review by the editorial office of this journal.

\section{Discussion}

Cardiac echinococcosis is commonly associated with hydatid disease in other organs (9), such as liver, lung, etc. The cardiac echinococcosis mostly invades the left ventricle (50-60\%), while septal wall (10-20\%), right ventricle $(5-15 \%)$ and pericardium (10-15\%) can also be affected (10-12). The high density and contraction of myocardium 
Table 1 Timeline

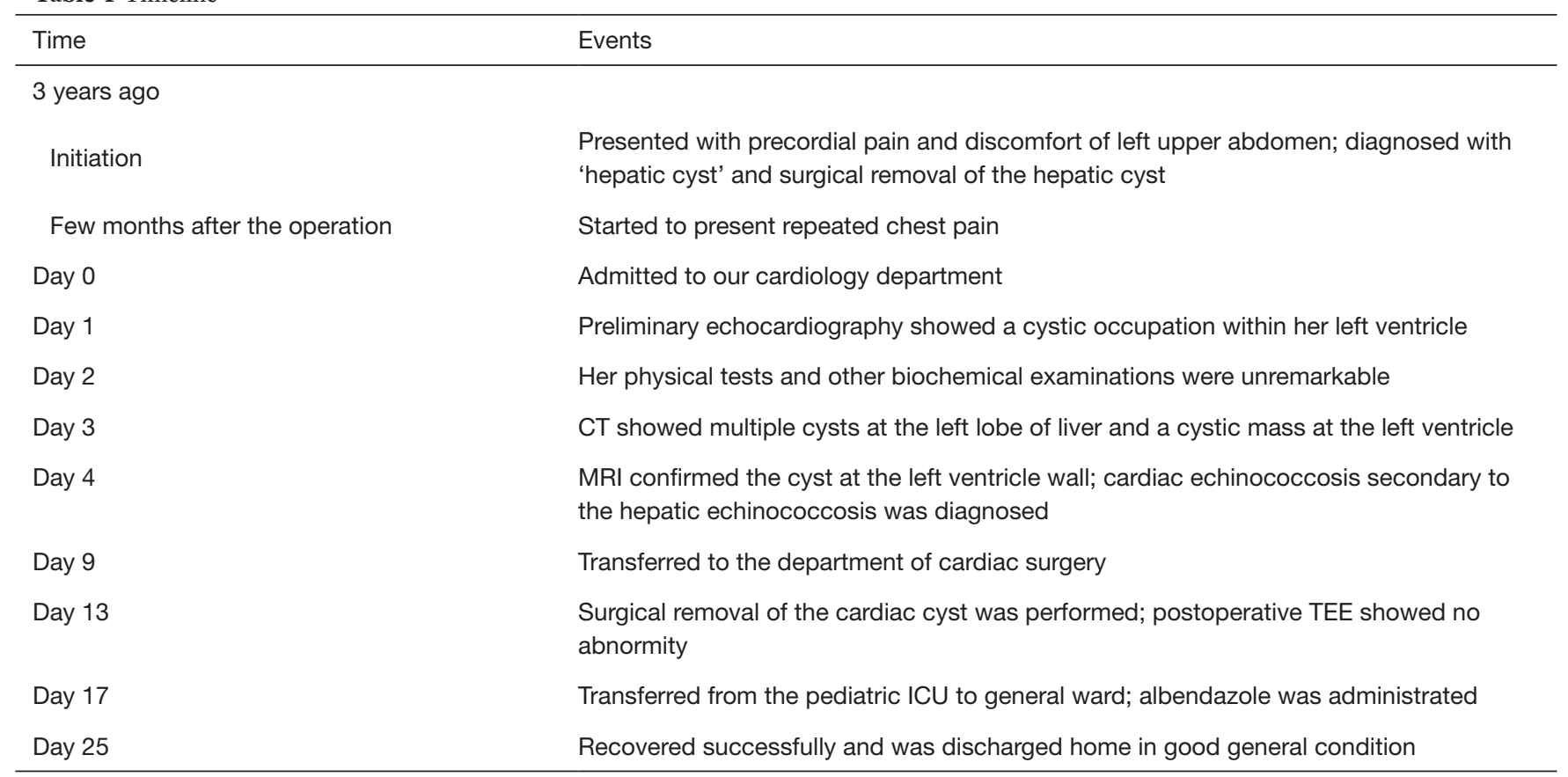

CT, computed tomography; MRI, magnetic resonance imaging; TEE, transesophageal echocardiography; ICU, intensive care units.

are not conducive to the survival of hydatid, hence, the cysts of cardiac echinococcosis are usually small and intracardiac rupture are of frequent occurrence.

Intact intramyocardial single hydatid cyst with a diameter less than $1 \mathrm{~cm}$ rarely cause symptoms and signs, thus infected patient may be asymptomatic for years. The cardiac echinococcosis is usually found after diagnosis of hydatid cysts within other organs during systematic examination (13). The symptoms are related to several factors such as location of cysts, number of cysts, or fistula formation. When the hydatid cyst in the myocardium develops to a large size, symptoms may show up such as shortness of breath, palpitation, and fever (14). Potentially life-threatening events such as cardiac tamponade, heart failure, syncope, arrhythmia, valvular stenosis or regurgitation, pulmonary hypertension, or peripheral embolism may occur as well (15). Hemoptysis in an indication when pulmonary echinococcosis is complicated. Sometimes hydatid cysts can block the ventricular outflow tract or invade the papillary muscles, resulting in valve stenosis or insufficiency, thus the corresponding cardiac murmurs can be heard in physical examination. If the cyst compresses the pulmonary trunk, jet systolic murmur can be heard. A few patients have allergic phenomena such as rash, measles or itching, which may be a precursor of serious complications such as fistula formation or rupture that requires special attention (16). Most of the symptoms or signs are similar with those of other cardiovascular diseases, thus increase the difficulty in reaching the correct diagnosis.

Hydatid disease is endemic worldwide, including the Mediterranean area, Balcanic nations, North and East Africa, India, China, Indonesia, and the Southern Cone of the Americas. In Tibetan regions of China, human CE is highly epidemic (17). This patient is a 13 -year-old girl who was raise in Tibetan plateau area. Precordial pain is the most obvious symptom, but her troponin and ECG examination were normal, thus the repeated chest pain could not be related to coronary heart disease but an influence of cyst on coronary blood flow. The $\mathrm{CE}$ is extremely rare since the improvement of people's life quality, therefore, the diagnosis of CE might be ignored by doctors. Residence in epizootic areas, occupations related to animal husbandry, and hydatid cysts in liver, lung or other extra-cardiac organs are all important clues for precise diagnosis of cardiac echinococcosis.

Since there are no characteristic symptoms and signs of cardiac echinococcosis, it is impossible to make a diagnosis just according to the clinical manifestations. Definite diagnosis of cardiac CE mainly dependent on imaging techniques. The most common applied imaging techniques 
include echocardiography, MRI, CT or X-ray, for detection of characteristic space-occupying intracardiac cysts (18). MRI can provide highly specific features of cardiac cysts, but it is expensive and not available in many underdeveloped CE endemic countries. Echocardiography is accessible, less expensive, and can identify pathological type of hydatid cysts, thus it is applied widely for precise diagnosis and postoperative follow-up.

The early surgical resection of cardiac cysts is indispensable due to the insidious progression and dangerous complications. Surgical treatment is generally feasible when there is no rupture or suppurative infection. The aim of surgical treatment is to avoid death caused by allergic reaction or heart failure. The effectiveness of surgery treatment depends on the number, location and sizes of cysts, as well as the complications. Surgical removal is usually performed when the heart is beating under the support of extracorporeal circulation machine. When puncturing and aspirating the contents of the capsule, standard preventive measures should be taken to avoid dissemination. In most cases, postoperative complications are rare, and recurrence is also uncommon, but if cardiac cysts are not treated timely and adequately, the prognosis of cardiac echinococcosis can be observably poor. It has been reported that the early mortality rate is about $10 \%$ (19). Albendazole therapy is the primary choice of CE treatment, but unfortunately, there is no unified standard of the course and dose of perioperative drug administration. Some previous literatures claimed that $\mathrm{CE}$ resection surgery should be performed after albendazole $10-15 \mathrm{mg} / \mathrm{kg} / \mathrm{d}$ is given preoperatively and the specific IgG antibody titer of hydatid is undetectable in serum (20). It has also been mentioned that surgical removal should be performed as early as possible, and albendazole should be given for a 3to 6-month-long course of treatment after surgery in order to prevent the recurrence and secondary infections (21). If the cardiac echinococcosis is combined with other organ infections, surgery or drug treatment should also be considered according to different conditions. This patient was supposed to have surgical removal of her hepatic echinococcosis cysts 3 months after the cardiac surgery, unfortunately, she did not back to our hospital within the required time and we lost the follow-up since the change of contact information. Therefore, the follow-up information, the therapeutic efficiency of surgery and Albendazole, and long-term prognosis were unclear.

In conclusion, cardiac echinococcosis is extremely rare in modern life, especially in children, which results in difficulty of diagnosis for clinicians. Echinococcosis must always be kept in mind when an intracardiac cystic image is detected even in early childhood as shown in this report. The combination of previous history and imaging techniques are important for making a definite diagnosis. Considering of the fatal complications and poor prognosis, it is important to make an early and precise diagnosis, and surgery should be performed as soon as possible before the complications occur. Besides, the early application of anti-hydatid drugs is also indispensable for cardiac echinococcosis treatment.

\section{Acknowledgments}

We would like to thank Dr. Bo Zhang for his help in polishing our paper.

Funding: This work was supported by the National Natural Science Foundation of China (No. 82071735); the R\&D Program for International S\&T Cooperation and the Exchanges of Chengdu (No. 2020-GH02-00068HZ) and Sichuan Science and Technology Program (No. 2021YFS0333).

\section{Footnote}

Reporting Checklist: The authors have completed the CARE reporting checklist. Available at https://cdt.amegroups.com/ article/view/10.21037/cdt-21-569/rc

Conflicts of Interest: All authors have completed the ICMJE uniform disclosure form (available at https://cdt.amegroups. com/article/view/https://dx.doi.org/10.21037/cdt-21-569/coif). The authors have no conflicts of interest to declare.

Ethical Statement: The authors are accountable for all aspects of the work in ensuring that questions related to the accuracy or integrity of any part of the work are appropriately investigated and resolved. All procedures performed in this study were in accordance with the ethical standards of the institutional and national research committee and with the Helsinki Declaration (as revised in 2013). Written informed consent was obtained from the patient for publication of this case report and accompanying images. A copy of the written consent is available for review by the editorial office of this journal.

Open Access Statement: This is an Open Access article distributed in accordance with the Creative Commons Attribution-NonCommercial-NoDerivs 4.0 International 
License (CC BY-NC-ND 4.0), which permits the noncommercial replication and distribution of the article with the strict proviso that no changes or edits are made and the original work is properly cited (including links to both the formal publication through the relevant DOI and the license). See: https://creativecommons.org/licenses/by-nc-nd/4.0/.

\section{References}

1. Jenkins DJ, Romig T, Thompson RC. Emergence/reemergence of echinococcus spp-a global update. Int J Parasitol 2005;35:1205-19.

2. Polat P, Kantarci M, Alper F, et al. Hydatid disease from head to toe. Radiographics 2003;23:475-94; quiz 536-7.

3. Atilgan D, Kudat H, Tükek T, et al. Role of transesophageal echocardiography in diagnosis and management of cardiac hydatid cyst: report of three cases and review of the literature. J Am Soc Echocardiogr 2002;15:271-4.

4. Kaplan M, Demirtas M, Cimen S, et al. Cardiac hydatid cysts with intracavitary expansion. Ann Thorac Surg 2001;71:1587-90.

5. Fiengo L, Bucci F, Giannotti D, et al. Giant cardiac hydatid cyst in children: case report and review of the literature. Clin Med Insights Case Rep 2014;7:111-6.

6. Nunes MC, Guimarães Júnior MH, Diamantino AC, et al. Cardiac manifestations of parasitic diseases. Heart 2017;103:651-8.

7. Yaliniz H, Tokcan A, Salih OK, et al. Surgical treatment of cardiac hydatid disease: A report of 7 cases. Tex Heart Inst J 2006;33:333-9.

8. Maroto LC, Carrascal Y, López MJ, et al. Hydatid cyst of the interventricular septum in a 3.5-year-old child. Ann Thorac Surg 1998;66:2110-1.

9. Birincioğlu CL, Bardakci H, Küçüker SA, et al. A clinical dilemma: cardiac and pericardiac echinococcosis. Ann Thorac Surg 1999;68:1290-4.

10. Abid A, Ben Omrane S, Kaouel K, et al. Intracavitary

Cite this article as: Zhang R, Shen C, Rao L. Cardiac echinococcosis secondary to hepatic echinococcosis: a rare case report. Cardiovasc Diagn Ther 2022;12(1):147-152. doi: $10.21037 / \mathrm{cdt}-21-569$ cardiac hydatid cyst. Cardiovasc Surg 2003;11:521-5.

11. Ozer N, Aytemir K, Kuru G, et al. Hydatid cyst of the heart as a rare cause of embolization: report of 5 cases and review of published reports. J Am Soc Echocardiogr 2001;14:299-302.

12. Wadhawa V, Shah J, Doshi C, et al. Surgical overview of cardiac echinococcosis: a rare entity. Interact Cardiovasc Thorac Surg 2018;27:191-7.

13. Rey M, Alfonso F, Torrecilla EG, et al. Diagnostic value of two-dimensional echocardiography in cardiac hydatid disease. Eur Heart J 1991;12:1300-7.

14. Miralles A, Bracamonte L, Pavie A, et al. Cardiac echinococcosis. Surgical treatment and results. J Thorac Cardiovasc Surg 1994;107:184-90.

15. Firouzi A, Neshati Pir Borj M, Alizadeh Ghavidel A. Cardiac hydatid cyst: a rare presentation of echinococcal infection. J Cardiovasc Thorac Res 2019;11:75-7.

16. Perez-Gomez F, Duran H, Tamames S, et al. Cardiac echinococcosis: clinical picture and complications. $\mathrm{Br}$ Heart J 1973;35:1326-31.

17. Li T, Chen X, Zhen R, et al. Widespread co-endemicity of human cystic and alveolar echinococcosis on the eastern Tibetan Plateau, northwest Sichuan/southeast Qinghai, China. Acta Trop 2010;113:248-56.

18. Li T, Ito A, Pengcuo R, et al. Post-treatment followup study of abdominal cystic echinococcosis in tibetan communities of northwest Sichuan Province, China. PLoS Negl Trop Dis 2011;5:e1364.

19. Sinha A, Nanda NC, Panwar RB, et al. Live threedimensional transthoracic echocardiographic assessment of left ventricular hydatid cyst. Echocardiography 2004;21:699-705.

20. Molavipour A, Javan H, Moghaddam AA, et al. Combined medical and surgical treatment of intracardiac hydatid cysts in 11 patients. J Card Surg 2010;25:143-6.

21. McManus DP, Zhang W, Li J, et al. Echinococcosis. Lancet 2003;362:1295-304. 